\title{
A Collaboration Between a Historically Black University and an Ivy League Psychiatric Research Center: A Psychiatrist's Reflections of the Impact on Residency Training
}

\author{
Mansoor Malik
}

Published online: 19 April 2013

(C) Springer Science+Business Media New York 2013

As detailed in the preceding paper, a central aim of the collaboration between our two institutions is building research capacity in minority-particularly, AfricanAmerican-mental health. Building capacity is at the heart of my role in the collaboration, specifically, and at my university more broadly. As the training director of the psychiatry residency program, I am responsible for coordinating training for a small but very vibrant group of residents. Most of our residents are minority or international medical graduates; therefore, cultural competency has assumed an ever increasing role in our curriculum. In accordance with the our university's mission, we take care to select residents who are sympathetic and possibly committed to the cause of reducing racial disparities in mental health.

Our university, being the largest historically Black university in the country, has played a unique role in efforts to reduce disparities by training and promoting minority health care researchers. Our university hospital was built on the foundations of Freedmen's Hospital and Asylum that cared for freed, disabled, and aged blacks. In 1868, after the Civil War, Freedmen's Hospital became the teaching hospital of our university medical school. In 1909, a new 278-bed Freedmen's Hospital was erected. Finally in 1975, the current and modern University Hospital was opened. Since that time the university had a small but thriving psychiatry department and is one of the main sites for African-American and other minority candidates to get residency training in psychiatry. The Psychiatry Residency Program was established in 1955 by a visionary Afro-Caribbean psychiatrist E.Y. Williams and is one of the oldest psychiatry training programs in the DC area. The current chair is a well-known researcher in the area of health disparities.

There is debate whether "race-based institutions of higher learning" like ours should exist in this day and age. Some consider them completely unnecessary and

M. Malik $(\bowtie)$

Department of Psychiatry, Howard University, Washington, DC, USA

e-mail: mamalik@howard.edu 
refer to them as an "expensive, ineffective anachronism" (Vedder 2010). Another hot issue relating to these institutions is the amount of subsidies such institutions receive. For example, our university receives about $\$ 235$ million from the federal government annually. Many argue that this subsidy should be given directly to the African-American students to attend other institutions. I confess that before moving to this institution, I shared some of the same criticisms. However, after working here for many years, I have acquired a great respect and admiration for such institutions. First, I did not realize how diverse a place like our university can be. There are not only African-Americans, but also a large student population of Africans, and students of Afro-Caribbean and Asian origin. At the University Hospital, at least half the residents are non-African-American. Although we look for residents with an interest in minority heath issues, the race of the candidates is not a deciding factor at all in selecting our residents. Through personal stories of many of the AfricanAmerican residents and faculty members at our university, I have come to appreciate their struggles. The university accepts many students that come from exceedingly poor black neighborhoods with substandard schools and poor scores on standardized tests. These students may not stand a chance to get into mainstream universities.

Our university and other historically black colleges and universities (HBCUs) have a crucial role to play in reducing racial disparities in mental health training and research. Although African-Americans and Hispanics compose over one-quarter of the U.S. population and over $11 \%$ of psychiatric residents, fewer than $6 \%$ of all applicants for NIMH funding and fewer than $4 \%$ of all applicants receiving such funding came from those minorities (National Advisory Mental Health Council Workgroup on Racial/Ethnic Diversity in Research Training and Health Disparities Research 2001). In a well-publicized NIH funded study, Ginter and colleagues found that Asians are $4 \%$ points and Black or African-American applicants are $13 \%$ points less likely to receive $\mathrm{NIH}$ investigator-initiated research funding compared to Whites. After controlling for the applicant's educational background, country of origin, training, previous research awards, publication record, and employer characteristics, Black applicants still remain $10 \%$ points less likely than Whites to be awarded NIH research funding (Ginther et al. 2011).

These discouraging statistics not only warrant policy interventions, but also highlight the need for HBCUs to be more proactive in pursuing health care research opportunities. Given the fact that almost $29 \%$ of African-American medical school graduates attended HBCU institutions for residency from 1950 to 1998 (IOM 2001), it is imperative that our university and other HBCUs develop and promote their research infrastructure to insure an adequate supply of minority mental health researchers. These researchers can, in turn, serve as role models to students and trainees, thereby increasing the "pipeline" of minority mental health researchers over time. My role in the Collaboration stems from this concern and involves promoting research education and participation among our psychiatry residents.

My perspective on psychiatric training and my commitment to reducing inequalities in mental health care are shaped by own unique experiences as an immigrant physician. I grew up in Pakistan. While growing up, I never encountered a Black population that was segregated and came with the baggage of slavery. 
However, there are religious minorities in Pakistan, such as Christians, Hindus, and other Muslim sects that are treated much the same way with the associated stigma. I came from such a Muslim sect called Ahmadyyia that continues to be persecuted in Pakistan. People from that group are forbidden by law to call themselves Muslims and are considered heretics. Sometimes they are attacked and even killed on the injunctions of Mullahs. I grew up with a sense of belonging to a small tightly-knit community. However, unlike African-Americans, my community had a higher educational and economic success rate than the general population. As the prospects of government jobs were all but closed to the members of our community because of their religious beliefs, the only way to be successful was to be entrepreneurial. We were driven to work extra hard and achieve educational success because of the hurdles we may face later in the life. The assumption was not to rely on the government or society at large for anything. Years later, during my medical training in the US, I heard that sentiment echoed by many of my African-American patients. I think the decision to pursue medical school and ultimately psychiatry was driven by this minority mind set.

My first encounter with a Black population was in the UK where I was trained in the NHS system. The Black population in the UK is mainly of Afro-Caribbean origin and is one of the largest minority groups, in addition to the South-Asian immigrant population. In the UK, for the first time, I found myself belonging to a minority group ("South Asians") that was both educationally and economically disenfranchised from the mainstream British population. South Asians fare marginally better than the Black population in the UK and despite efforts aimed at integration, these groups mainly live in segregated neighborhoods. Derogatory slangs and public hostility were fairly common during the mid-1990s, when I was there. One night, while working in the emergency room, one of my patients referred to me as "Paki" and told me that I should go back to the "hole I came from." When I told my attending physician, referred to as consultants in the UK, she told me to just "brush it off."

In my British training, there was little emphasis on cultural training or understanding. The bent of training was decidedly "biological." Psychiatric disorders were looked at from a Western perspective and any alternatives were considered little more than superstition. Certainly, the situation has much improved since. Perhaps as a rebellion against this narrow approach, I started to develop a greater interest in anthropological and cultural aspects of psychiatry. My first introduction to this unchartered territory was Erving Goffman's "Asylums: Essays on the Social Situation of Mental Patients and Other Inmates." I was equally fascinated by Thomas Szasz and R.D. Laing. This was the first time I fully realized that psychiatry is not the "biomedical" science I was led to believe it was. I must confess that the uncertainty about the intellectual foundations of my chosen profession was quite disconcerting and led to significant soul searching.

After some hesitation about whether to go back to Pakistan, I decided to come to the US for further training, mainly for family reasons. My first stop was Philadelphia. I started working in an inner city hospital with a predominantly African-American population. Nothing could have prepared me for the shock I felt when I first realized the grinding realities of my patients' lives. I had a very different 
view of American society and did not expect to see this abject poverty, rampant drug use, severe lack of educational and recreational facilities, the absence of fathers in the family structure and overwhelming difficulties of single mothers trapped in grueling work routines.

One night, while on call, I received a distressing phone call from one of the clinic patients, who was a 30-year-old single mother of five. She worked as a janitress, did not get any support from the three men who had fathered her children, and had long history of depression and alcohol dependence. She nervously informed me that out of her five young children, one was threatening to set the house on fire, another one had jumped out of the window and the third one was trying to attack the others with a knife. I had her admitted to the psychiatry inpatient unit. All of her children were already in treatment and on medications for ADD and oppositional defiant disorder. When I presented the case to the attending psychiatrist the following day, he advised me to call the child protective services so the children would be "safe and protected" and to double the antidepressant dose for my patient. I did not feel that my Jewish White attending, who was a successful analyst with a busy practice, had any understanding about my patient's life experiences, her strong desire to move her children out of the housing project and to keep them from going into foster care system. I wondered, would we treat this patient the same way if she had been an attorney or physician from a wealthy background?

After completing my residency, I went to Dartmouth for a fellowship. Dartmouth was a world apart from inner city Philadelphia. This was a culture of economic stability, if not wealth and confidence, if not entitlement. My patients were mostly sophisticated and educated white collar workers. Somehow I did not feel as "needed" there and, after finishing my training, took the opportunity to move to Washington DC.

As an immigrant physician, I was impressed by our university's openness to train medical graduates from other countries, the so-called "International Medical Graduates" (IMGs). Sponsoring and training foreign medical graduates requires a lot of extra work in addition to expensive visa processing and legal fees. Therefore, many institutions (especially the more elite ones) do not accept foreign medical graduates at all. Although IMGs constitute a full $30 \%$ of the psychiatry work force nationwide (Smart 2011), very few residency programs have acculturative and cultural competency programs that specifically take the needs of IMGs into account. However, at our university there is enthusiastic institutional support to address the unique issues related to the psychiatric training of IMGs such as language obstacles, reaction to immigration and reconciling cultural values.

In the pages to follow, I reflect on the ongoing research and training collaboration and, particularly, the goal of capacity building and professional development for minority mental health researchers. I hope to provide an assessment of both the opportunities and the challenges posed by this collaboration.

Every fall, a group of psychiatry residents travel to our partnering psychiatric research center to attend a multi-day seminar on Evidence-Based Medicine taught by faculty and biomedical librarians. Some of our residents, especially those in their 4th year, have already had an elective research rotation and have substantial experience using search engines such as PubMed and are familiar with the 
MEDLINE and Cochrane Databases. Others are just learning the evidence-based paradigm. Similarly, some residents readily embrace the use of numeric tools in appraising the evidence, whereas others are less comfortable with statistics and mathematical operations. A special feature of the seminar is that it is tailored to the individual level of skill and comfort of the residents.

Care is taken to provide a personalized learning environment by providing case material relating to the residents' clinical interests. For example, in 2010, we selected a large randomized controlled trial for treatment resistant schizophrenia and a meta-analysis on the risk of death with the use of antipsychotics in dementia. Both of these topics were very relevant to the residency training and were clinically familiar to the residents. Residents welcomed the opportunity to apply evidencebased techniques to familiar clinical situations and appreciated how newly acquired skills could be directly relevant to their day-to-day practice. The next year, we expanded this approach and the residents were asked to use their Evidence-Based Medicine skills to address a clinical case that they prepared prior to coming to the Institute. This last step served as a direct bridge between the seminar and the residents' clinical practice and the challenges of their clients.

Our residents also participate in the Collaborative Summer School, which is an annual, week-long, academic research training program (see Hipolito et al. 2012 for a fuller description). Although not geared primarily toward resident education, it is an invaluable opportunity for residents to acquire tools needed to become better researchers, clinicians, and scholars, especially as related to African-American mental health. However, residents' involvement in the summer school is limited by their clinical responsibilities, which make it impossible to attend and participate in the full week of seminars and activities. Typically, residents participate en masse on their "didactic day," the only day of the week devoted purely to research and training. Thus, while the residents enjoy—and, I believe, truly benefit from-the summer school, we are missing opportunities for more meaningful engagement by not working together to mitigate scheduling conflicts in recognition of the time constraints and serious pressures under which our residents operate. Our hope is that trainees and potential mentors maintain regular communication following the summer school. Having more time to network with potential mentors and discuss clinical research opportunities would strengthen the impact of the summer school for the residents.

Lastly, through the collaboration, the psychiatry residents have been introduced to anthropological research methods by becoming involved with the ongoing collaborative research projects. Anthropological approaches to mental illness and recovery are new to most of our residents. For most, the seeming informality of ethnographic and qualitative techniques has been challenging to reconcile with their training in the "hard" sciences. The Department of Psychiatry at our university, like most contemporary psychiatry department has a strong bias toward biological psychiatry. Residents also felt uncomfortable with the informal teaching style of the visiting social scientists, which went against the hierarchical culture at our university. Our efforts to introduce residents to much wider philosophical approach to psychiatry were viewed as a distraction from the core learning of the so-called biological psychiatry. Our efforts to incorporate anthropological and sociological 
topics in resident curriculum were met with skepticism by senior faculty and many residents themselves. This was compounded by the fact the residency program had lost its previous program director and I, as the new training director, was just coming onboard.

Over time, we learned that the residents' apparent unease with the Kleinmanian approach of seeking "what really matters" to people was rooted in dynamics internal to the residency program. In short, the residents felt that what mattered to them was irrelevant insofar as the training structure was concerned because no one listened to them anyways. It is interesting to note that three residents chose to transfer to other programs during this time, no doubt influenced by many other factors as well. Perceiving that introduction of anthropological methods of inquiry was uncovering the latent anxieties about power sharing and hierarchy within the residency program, we introduced a regular confidential process group run by an outside contracted psychologist (not a faculty member). Gradually, with more didactic sessions and more informal interactions with the faculty of our partnering research center, residents felt more comfortable expressing their opinions and sharing their concerns. In addition, the residents have come to appreciate anthropological methods and theory that place disparity research in the context of social justice and promote a sophisticated understanding of "culture" and its role in disparities.

Not unexpectedly, the experience with the collaboration has led to a greater discussion of resident acculturation and cultural competency issues. The residents have become much more open in communicating their observations about cultural differences. For example, one of the residents, originally from India, was having difficulty in eliciting a full psychiatric workup while on call. She shared with me that, in India, patients use self-disclosure as a way to establish a deeper sense of connection with the physician, whereas in US, it is a prerequisite for comprehensive history taking, without connotation of a deeper connection. Therefore, she felt uneasy about asking too many questions during the initial work up.

Although the overall impact of the collaboration has been extremely positive, it has not been without its challenges. One particularly upsetting incident occurred when residents were asked to submit small research proposals with a promise for funding through the collaboration grant. Residents spent a lot of time thinking about these projects and tried to carefully think through issues of study design and methods. Ultimately, six proposals were submitted, an impressive number for a small program of 16 residents. Residents had a chance to discuss their proposals with faculty from our partner research center during their training workshop in fall and genuinely felt excited about the opportunity. Yet this swell of excitement was soon dashed when the fate of these projects was decided in a meeting in early spring at our university. Unfortunately, a proposal from a senior faculty member from our university was selected for funding without any discussion of the residents' proposals at all. Residents viewed this as the reassertion of the hierarchical authoritative model and despite subsequent efforts to engage them, their participation in research activities with the collaborative projects remained low.

Although research capacity building has been an expressed goal of the Collaboration, this unfortunate incident illuminates how more junior researchers, and psychiatry residents in particular, have struggled, at times, to meaningfully 
participate in research activities that would further their professional development. The residents I have supervised are eager and excited by the opportunities posed by our collaboration. Their participation in the annual evidence-based medicine workshop stands out as a very meaningful training opportunity. Still, the potential of our residents has not been fully realized in the collaboration. It appears that most of the resources have been used by senior level faculty, already well established in their research careers. Also the collaboration failed to envision a plan to incorporate residents as junior researchers from the outset, and this has only emerged in piecemeal fashion. Nonetheless, there has been a substantial increase in resident driven abstracts and peer-reviewed publication at our university. As early exposure to research opportunities is one of the few correlates to a sustained research career (Goldman 2001), our hope is that the Collaboration will stimulate our residents to become successful clinician educators. Finally, we hope that this collaboration will be a model for other HBCU institutions. Greater involvement of underrepresented minorities is imperative if psychiatry is to offer the most responsive care to a diverse U.S. population.

\section{References}

Ginther, Donna K., Walter T. Schaffer, Joshua Schnell, Beth Masimore, Faye Liu, Laurel L. Haak, and Raynard Kington

2011 Race, Ethnicity, and NIH Research Awards. Science 333: 1015-1019.

Goldman, C., and V. Williams

2001 Benefits and Feasibility of a Conceptual Framework for Biomedical and Behavioral Science Personnel. Arlington, VA: RAND.

Hipolito, Maria, Mansoor Malik, Elizabeth Carpenter-Song, and Rob Whitley

2012 Capacity Building for African American Mental Health Training and Research: Lessons from the Howard-Dartmouth Collaborative Summer School. Academic Psychiatry 36(1): 47-50.

Institute of Medicine

2001 The Right Thing to Do, the Smart Thing to Do: Enhancing Diversity in Health ProfessionsSummary of the Symposium on Diversity in Health Professions in Honor of Herbert W. Nickens, M.D. Washington, D.C: National Academy Press.

National Advisory Mental Health Council Workgroup on Racial/Ethnic Diversity in Research Training and Health Disparities Research

2001 An Investment in America's Future: Racial/Ethnic Diversity in Mental Health Research Careers. Washington, DC: National Advisory Mental Health Council Workgroup on Racial/Ethnic Diversity in Research Training and Health Disparities Research.

Smart, D.R.

2011 Physician Characteristics and Distribution in the U.S. Chicago: American Medical Association Press.

Vedder, Richard

2010 Why Do We Have HBCUs? The Chronicle of Higher Education blog, http://chronicle.com/ blogs/innovations/why-do-we-have-hbcus/27506, accessed August 10, 2012. 